\title{
Abuse liability and the regulatory control of therapeutic drugs: untested assumptions
}

\author{
James H. Woods \\ Department of Pharmacology, University of Michigan Medical School, Ann Arbor, MI 48109-0010 (U.S.A.)
}

The drug abuse crisis now apparent in the U.S. has raised questions about some of our oldest and most fundamental assumptions regarding the effects of our systems for controlling the availability of drugs of abuse. Indeed, we have been compelled to reconsider whether we might not be better off without our venerable legal sanctions against these substances, especially when the clearest effects of these laws has been to make illegal commerce in these drugs so competitive as to imperil the safety of our streets and neighborhoods and so lucrative as to corrupt and destabilize the governments of whole nations.

In the case of drugs with abuse liability that also have significant medical value, the issues of regulatory control, though less dramatic, may be even more difficult: Here the aim of societal controls is not only to restrict the availability of these substances for abuse, but to do so without restricting their appropriate medical use. However, we generally assume that the regulations by which we choose to safeguard society will have the intended effects; and we assume no less of the regulations we apply to therapeutic drugs. This assumption has by and large gone unexamined and untested.

In fact, considering the history of these regulations on both the national and international levels, it is not clear that they have reduced the abuse of these drugs more than their appropriate use. Indeed, it is a telling commentary on the current state of affairs that one can question whether such regulations have had any effect on abuse of these drugs at all $[1,2]$. On the other hand, there is some evidence that societal controls have led to serious restrictions in the therapeutic use of some drugs, and thus to unnecessary suffering.

Perhaps the best case in point is that of narcotic analgesics, which first came under control in the U.S. in 1914 because of their "addictive" properties. It was noted as early as 1918 that these controls deterred medical use of these drugs [3]. More recent studies have consistently found that physicians managing pain tend to underuse these medications, apparently because of fears of inducing dependence - so that a substantial proportion or even the majority of hospital patients requiring analgesia continue to suffer moderate to excruciating pain $[4-8]$. What is the purpose of this suffering, when the actual risk of 'addiction' among such patients is very slight - possibly less than $0.01 \%[9,10]$.

We must reassert the need to practice medicine on the basis of science, rather than to be governed by societal pressures inspired by fears of 'addiction'. The challenge to research is to provide a clearer view of the phenomena of physiological and psychological dependence and of their social and clinical significance; and to better qualify the meaning of drugs' abuse liability in terms of the relative risks they pose for patients and for 
others. An overlapping objective is to begin to test the assumptions underlying regulation of therapeutic drugs with abuse liability, and to elucidate ways in which these regulations or their implementation may be improved.

There are a great many research needs in this area. The paramount needs are to develop sensitive and reliable measures of the consequences of drug regulation; and to increase the frequency, regularity, and scope of efforts to examine these effects. The goal is to establish ongoing monitoring systems that can provide an accumulation of experience to guide future policy-making.

\section{An illustrative case: regulation of benzodi- azepines}

It is instructive to consider these issues in the context of public policy governing the use of the benzodiazepines. These drugs have long been established as effective in therapy for anxiety and insomnia and are the agents most commonly used in treating these disorders; because of their role in treatment of musculoskeletal and convulsive disorders, they are classified as 'essential' drugs by the World Health Organization. The sheer volume of information available from research on the use and abuse of this group of drugs is probably greater than that for any other drug class. It is interesting to inquire how well this body of information has served, and how it might serve better, as a basis for regulation of these compounds.

\section{Information relevant to regulation}

Numerous studies of prescribing behavior and of actual drug use in the community indicate that the vast majority of use of benzodiazepines is appropriate with respect to the patients and conditions treated [11]. Epidemiologic research has also indicated that, at least in the U.S., the majority of people suffering high levels of psychic distress [12], or diagnosable anxiety syndromes [13], go without anxiolytic treatment. Thus the risk here is one of undertreatment [14]; it follows that particular caution is needed to avoid further restricting appropriate treatment for these problems.

What risks do the benzodiazepines pose? They are clearly safer in overdose than older sedatives/hypnotics, e.g. the barbiturates [15]. With regard to abuse, I will briefly summarize the findings of a recent review [11]: Although experimental models cannot be regarded as definitive predictors of abuse liability, the findings of experimental assessments of benzodiazepines' abuse liability have found remarkable parallels in epidemiologic research. That is, laboratory studies have demonstrated little or no reinforcing effects of benzodiazepines in normal subjects nor in subjects representative of typical patient populations; however, some preference for benzodiazepines has been demonstrated among subjects with histories of sedative abuse. Similarly, drug abuse surveys find little or no misuse or recreational use of benzodiazepines among the general population, but a greater prevalence of use of these drugs in various populations of abusers, including patients on methadone maintenance, opioid abusers, and, perhaps, alcoholics. Thus, abuse of benzodiazepines is limited to the relatively small population of individuals who tend to abuse multiple drugs. Clinical studies have shown that physiological dependence can develop in patients taking therapeutic doses of benzodiazepines over prolonged periods; however, this dependence does not appear to be accompanied by escalation of dose or drug-seeking behavior, which would characterize abuse.

Current regulation at the national and international levels

The principal effect of international control of the benzodiazepines, under Schedule IV of the Psychotropic Convention of the U.N., is that these drugs may be dispensed only upon medical prescription. In the U.S., benzodiazepines are also controlled under Schedule IV of the Controlled Substances Act, so that prescriptions can be refilled no 
more than five times within six months of the original prescription.

\section{Regulation by New York State}

However, consider the instance of a regulation imposed in January 1989, by New York State. Under this regulation, benzodiazepine prescriptions can now be issued only on the State's official triplicate prescription forms, of which one copy must be submitted to the Department of Health for purposes of computerized surveillance. For the most prevalent disorders for which these drugs are used, prescriptions are limited to a 30-day supply and cannot be refilled. This multiple-copy prescription program had previously been applied, in New York and other states, primarily to drugs listed in Schedule II of the federal Controlled Substances Act, i.e., drugs whose abuse can lead to 'severe psychological or physical dependence' (21 U.S.C. $812(\mathrm{~b}) 2(\mathrm{C})$ ). The ostensible reason for adopting this regulation was to reduce illicit traffic in and abuse of benzodiazepines. However, the evidence of abuse presented by the State was not compelling. For example, the State's argument [16] rested heavily on data from the Drug Abuse Warning Network (DAWN) pertaining to emergency room visits in which benzodiazepines were involved; in fact, an official analysis of DAWN data for 1976 - 1985 found that such visits significantly declined over this period, both for the U.S. as a whole and specifically for the New York State facilities reporting to the system [17]. Conspicuously absent from the State's argument was any attempt to describe how or whether this alleged abuse might relate to legitimate use of these drugs, as well as any attempt to indicate exactly how this specific regulatory action was supposed to reduce the types of abuse and other problems described.

\section{The implicit assumption about prevalence of use}

The underlying motivation for this regula- tion may have been revealed by the emphasis, in the State's argument [16], on the 'enormous' prevalence of use of the benzodiazepines. The implicit assumption was that this widespread use alone must be evidence of some problem, e.g., overprescription or abuse. If the prevalence of drug use alone should raise suspicions, why did New York choose to act against the benzodiazepines rather than, say, beta blockers or antiinflammatory agents, which are even more widely used?

Conspicuously absent from this part of the State's argument was any attempt to relate the prevalence of use of these drugs to the prevalence of the health problems for which their use is indicated. As I have argued previously [11], the prevalence of use of sedative/hypnotic medications has not changed appreciably over the period since the time of the earliest relevant records - i.e., for at least the better part of a century. It seems reasonable to assume that the need for such drugs is related to the prevalence of the illnesses for which they are used, which affect about 15 to $20 \%$ of virtually any population studied.

In states where multiple-copy prescription programs have been implemented, prescriptions for the affected drugs have declined by 30 to $55 \%$ in the programs' first two years [18]. Based on this experience, as well as the thrust of the New York State argument, it seems clear that the State intended not merely to curtail abuse of benzodiazepines but to reduce the overall prevalence of their use. Predictably, then, prescription audit data from New York State indicate that benzodiazepine prescriptions have already declined by about $50 \%$ [19]. Since, as described above, the best available information indicates that the vast majority of benzodiazepine prescriptions are appropriate, and that abuse is virtually limited to the relatively small population of multiple-drug abusers, the New York State regulation is clearly affecting medical use more than abuse of these drugs. 
Lessons about the role of research

If drug regulations are to safeguard the public health, what safeguards do we have against drug regulations that are expressions of prejudice rather than science? The distressingly inappropriate regulation imposed by New York State emphasizes the importance of building meaningful scientific accountability into the regulatory structure. The legislation under which the New York State Commissioner of Health acted gave him the power to impose this regulation with no obligation to assess the relevant scientific evidence nor to defend the merits of his decision on the basis of such evidence. This kind of drug legislation, providing no effective role for the findings of good research in the policy-making process, can lead to a form of authoritarian control that is not only inconsistent with good public health practice but which, as in this case, can actually jeopardize the public health.

Fortunately, many regulatory systems, particularly at the national and international levels, do at least require expert assessments of the available evidence as based for policy decisions. Even these systems are flawed, however, in that they do not mandate nor provide mechanisms for surveillance of the actual impact of drug regulation on abuse and on medical use of the drugs affected; without such surveillance there is no assurance that these regulations actually operate in the best interests of the public health.

Another important lesson from the New York State experience is that, with respect to therapeutic drugs, it is inappropriate to draw policy conclusions based on evidence of abuse alone. Such evidence rarely includes adequate characterization of the circumstances or patterns of abuse, or, most importantly, of the population at risk. As illustrated by the example of the benzodiazepines, abuse liability is not an intrinsic pharmacologic property of a drug or drug group, such that it necessarily places all populations at equal risk; rather, abuse liability can differ not only among individuals but also among populations and among environmental circumstances.

\section{Research needs}

Thus it is important to establish the relevance of evidence of abuse as a basis for regulatory policy. This requires research designed to specify the relative risks for different population subgroups. The most promising approach is epidemiologic research designed to examine the variety of ways in which a drug available to a population is actually used, i.e., examination of both use and abuse in the same population.

For example, studies of the use and abuse of benzodiazepines have included computerbased drug utilization reviews which focused on records of prescriptions dispensed within large medical complexes [20-22]; prospective studies of all individuals within a region who filled sedative/hypnotic prescriptions, which examined these individuals' subsequent prescription purchases over time [23-25]; and community surveys in which respondents were asked about medical and nonmedical use of various psychotropics [26].

It is research of this kind that can provide the information necessary to tailor regulations to the specific risks that drugs actually pose. In addition, the implementation of ongoing studies of this kind, i.e., studies that examine use and abuse in the same populations would enable us to monitor the effects of regulatory interventions, and to build a cumulative information base for use in formulation of drug policy.

In considering policy on drugs with substantial medical value and use, we should be concerned primarily with the potential effects on people who might benefit from treatment with these agents. If we are concerned about the risk of abuse of a given drug among these people, we should rely on studies of its liability for abuse in these people or their representatives. It is not reasonable to deprive a patient of a potentially beneficial medication in the interest of reduc- 
ing the risk that it might be abused by someone else.

\section{Conclusion}

If it is in the interest of the public health to require that drugs meet certain standards of safety and efficacy. It should be of equal concern that regulations affecting the availability of these drugs should prove safe and effective. A primary role for research, in addition to providing reliable information on the abuse liability of therapeutic drugs, should be to help establish standards for the safety and efficacy of regulatory interventions. When we apply regulations, we should have systems in place for monitoring whether they do have their intended effects, and for detecting and measuring the frequency of their unintended and unwanted effects.

As scientists, we should regard each instance in which a regulation is imposed on a therapeutic compound as an opportunity for evaluation of the effects of this interven tion: the intended effects on the behavior of prescribers, patients, and drug abusers, as well as an array of possible secondary effects. It is time that we learn to take advantage of these natural experiments, beginning by establishing appropriate measures of these effects.

As advisors on drug policy, we should ensure that these measures are carefully constructed and systematically applied, to provide an accumulation of experience on which to base regulations that will protect rather than jeopardize the public health.

\section{Acknowledgements}

I acknowledge with appreciation the comments on the paper by R. Griffiths, C. Johanson, E. Kaim and K. Warner.

\section{References}

1 F. Hoffmeister and G. Stille, Acta Neurochirurgica, 38 (1987) 44.
2 O. Ekeberg et al., Acta Med. Scand., 221 (1987) 483.

3 J.H. Jaffe, Drug Alcohol Depend., 14 (1985) 403.

4 R.M. Marks and E.J. Sachar, Ann. Intern. Med., 78 (1973) 173.

5 J.J. Bonica, Cancer pain. In: J.J. Bonica (Ed.), Pain, Raven Press, New York, 1980, pp. 335-362.

6 M. Angell, N. Engl. J. Med., 306 (1982) 98.

7 J.P. Morgan, Adv. Alcohol Subst. Abuse, 15 (1985) 163.

8 M. Donovan, P. Dillon and L. MeGuire, Pain, 30 (1987) 69.

9 J. Porter and H. Jick, N. Engl. J. Med., 302 (1980) 123.

10 W.T. Beaver, J. Am. Med. Assoc., 234 (1980) 2653.

11 J.H. Woods, J.L. Katz and G. Winger, Pharmacol. Rev., 39 (1987) 251.

12 G.D. Mellinger et al., Arch. Gen. Psychiatry, 35 (1978) 1045.

13 E.H. Uhlenhuth et al., Curr. Med. Res. Opinion, 8 (1984) 37.

14 E.H. Uhlenhuth et al., J. Clin. Psychopharmacol., 8 (1988) 161.

15 D.J. Greenblatt et al., Clin. Pharmacol. Ther., 21 (1977) 497.

16 J.L. Eadie, Affidavit in support of motion for summary judgment, Index No. 13910/87, submitted to the Supreme Court of the State of New York. County of New York, February 8, 1989.

17 A Decade of DAWN: Benzodiazepine-Related Cases, 1976-1985, Statistical Series H, Number 4, DHHS Publ. No. (ADM) 88-1575, U.S. Department of Health and Human Services, 1988.

18 Multiple Copy Prescription Programs Resource Guide, Drug Enforcement Administration, U.S. Department of Justice, 1987.

19 I.M.S. America Ltd., Ambler, Pennsylvania.

20 R.F. Maronde et al., Calif. Med., 117 (1972) 22.

21 R.F. Maronde and M. Silverman, Ann. Intern. Med., 79 (1973) 452.

22 J.J. Scrivens et al., Hosp. Formul., 14 (1979) 830.

23 G. Boethius and B. Westerholm, Acta Med. Scand., 199 (1976) 507.

24 G. Boethius and B. Westerholm, Acta Psychiatr. Scand., 56 (1977) 147.

25 G. Boethius, Prescription Pattern in 234 Individuals with First Prescription of Hypnotics/Sedatives in 1976: A 6-years' Follow-up, Second World Conference on Clinical Pharmacology and Therapeutics, Washington, D.C., July 1983.

26 G.D. Mellinger and M.B. Balter, Psychotherapeutic drugs: A current assessment of prevalence and patterns of use. In: J.P. Morgan and D.V. Kagan (Eds.), Society and Medication: Conflicting Signals for Prescribers and Patients. D.C. Heath and Co. Lexington, 1983, pp. $137-144$. 